\title{
Energy resolution at the Fano limit: The benefits of the principle of sideward deple- tion for solid state X-ray spectrometry
}

\author{
Julia Schmidt ${ }^{1}$, Jeffrey M. Davis ${ }^{1}$, Martin Huth ${ }^{1}$, Robert Hartmann², Heike Soltau ${ }^{1}$, Lothar Strüder ${ }^{2,3}$ \\ 1. PNDetector GmbH, Otto-Hahn-Ring 6, 81739 München, Germany \\ 2. PNSensor GmbH, Otto-Hahn-Ring 6, 81739 München, Germany \\ 3. University of Siegen, Walter-Flex-Strasse 3, 57072 Siegen, Germany
}

In the field of X-ray spectrometry, high-energy resolution and low-energy detection capability are important requirements. The energy resolution of a detector system depends on the intrinsic resolution of the detector chip and on the electronic noise of the system. The intrinsic resolution of the detector chip is based on statistical fluctuations of the signal conversion mechanism and defines a lower limit of the best achievable energy resolution, which is called the Fano limit. The noise, which is added by the readout electronics, depends on the detector characteristics, consisting of leakage current and output capacitance and on the characteristics of the front-end electronics. In order to improve the energy resolution, the detector capacitance and the stray capacitance connected to the output node should be as small as possible.

In 1984 E. Gatti and P. Rehak introduced a new detector scheme based on the principle of sideward depletion [1]. The concept is that a large semiconductor wafer of usually high resistivity n-type silicon can be fully depleted from a small $\mathrm{n}^{+}$ohmic contact which is positively biased with respect to the $\mathrm{p}^{+}$electrodes covering both sides of the wafer. According to this biasing mechanism, the electric potential perpendicular to the wafer surface has a parabolic shape, with a minimum depending on the potential difference over the wafer (Figure 1).

This talk will show the historical development and the benefits of sideward depletion devices for high performance spectroscopy and spectroscopic imaging. SDDs (silicon drift detectors) will be discussed, as well as pnCCDs (pn-charge coupled devices) and DEPFET (depleted field effect transistors) active pixel sensors.

The SDD is derived from the principle of sideward depletion by adding an electric field parallel to the wafer surface. This is achieved by a segmentation of either both or one of the $\mathrm{p}^{+}$areas at the surfaces to form strip patterns and by adding a voltage gradient to the strip patterns. The direction of the voltage gradient is such that the $\mathrm{n}^{+}$readout anode is the electron potential minimum and therefore collecting all signal electrons. The main advantage of the SDD compared to a standard PiN-diode of the same size is the small anode capacitance which is independent of the active area of the device. Since the electronic noise at short shaping times is proportional to the capacitance squared, the principle of sideward depletion results in a better energy resolution at shorter shaping times (higher count rates), in particular at low energies where the signal-to-noise ratio is lower. Moreover, the double sided design allows to build devices with a homogeneous entrance window optimized for low energy X-rays.

Another novel detector taking advantage of the principle of sideward depletion is the so-called pnCCD [2]. The pnCCD is an energy dispersive detector in which every pixel serves as a sort of SDD. Therefore, the pnCCD combines the energy resolution of an SDD with the spatial resolution of a CCD. The pnCCD is a backside illuminated, three-phase CCD in which the transfer registers are formed by pn-junctions, 
increasing the radiation hardness of the device. By biasing the diode at the entrance window negatively with respect to the substrate or anode, the electron potential minimum can be shifted closer to the side containing the pixel structure. Due to the charge transfer at a moderate distance from the surface, the pnCCD can be operated at high speed. Since the full volume is depleted, the pnCCD has an enhanced sensitivity compared to standard CCDs, which is especially important for measurements of X-rays with higher energy.

The DEPFET pixel detector is based on a combination of the method of sideward depletion and the principle of the field effect transistor [3]. As with the pnCCD, the potential minimum is moved close to the top side. A transistor is on top of each DEPFET pixel. The signal electrons are located in the potential minimum of the channel of the transistor (the internal gate) and increase the transistor channel conductivity by induction. The device can be reseted by applying a large positive voltage on the clear electrode. Therefore, the DEPFET combines the function of a sensor and an amplifier. It is fully sensitive over the complete bulk and it has a low capacitance and a low noise.

\section{References:}

[1] E Gatti and P Rehak, Nuclear Instruments and Methods in Physics Research 225 (1984), p. 608.

[2] L Strüder et al, Review of Scientific Instruments, 68 (1997), p. 4271.

[3] G Lutz et al, Sensors, 16 (2016), p. 608.

[4] G Lutz in "Semiconductor Radiation Detectors" (Springer Publishing, Heidelberg) p.126.

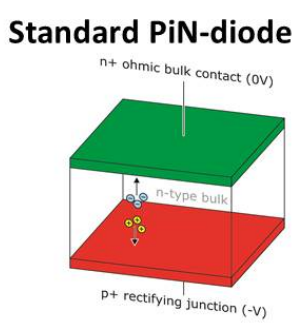

Double side $p$-junction with sideward $\mathrm{n}^{+}$contact

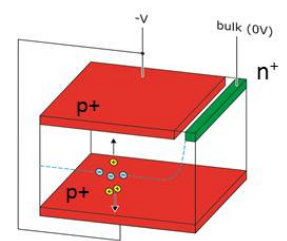

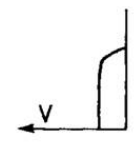

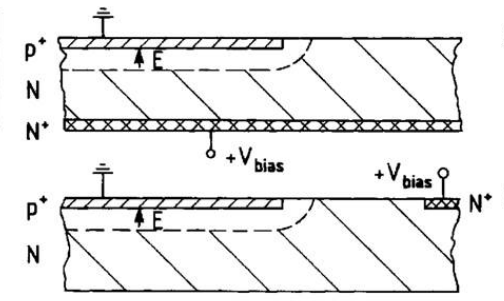

Standard PiN-diode

- Depletion by back bias

Sideward depleted detector

- Depletion from side bias
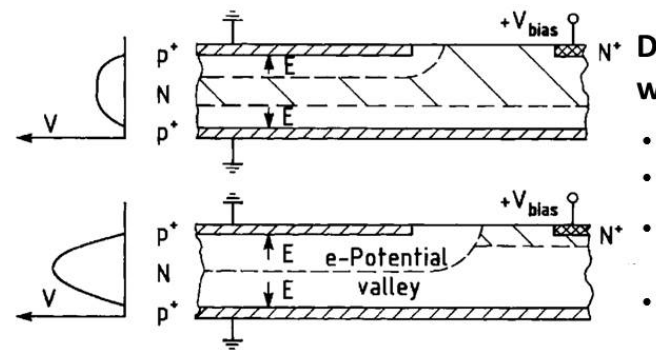
with sideward $\mathrm{n}^{+}$contact

- Moderate side bias

- Partially depleted detector

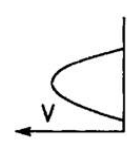

Increased side bias for fully depleted detector

Minimum for electrons depends on potential difference over the detector

Figure 1: Principle of sideward depletion. The semiconductor wafer is fully depleted through a small $\mathrm{n}^{+}$ contact reverse biased with respect to the $\mathrm{p}^{+}$implants realized on the two sides of the detector. The path of the charge carriers (left) and the electric potential (right) for different depletion geometries are shown. On the images on the right side, the hatched areas indicate the non-depleted sections. [4] 